\title{
A NOTE ON DIFFERENTIAL-ALGEBRAIC SYSTEMS WITH IMPULSIVE AND HYSTERESIS PHENOMENA
}

\author{
Pavel Petrenko \\ Matrosov Institute for \\ System Dynamics and \\ Control Theory of SB RAS \\ Russia \\ petrenko_p@mail.ru
}

\author{
Olga Samsonyuk \\ Matrosov Institute for \\ System Dynamics and \\ Control Theory of SB RAS \\ Russia \\ samsonyuk.olga@gmail.com
}

\author{
Maxim Staritsyn \\ Matrosov Institute for \\ System Dynamics and \\ Control Theory of SB RAS \\ Russia \\ starmaxmath@gmail.com
}

Article history:

Received 14.10.2019, Accepted 10.12.2019

\begin{abstract}
In this note, we single out some promising classes of differential-algebraic equations (DAEs) with hysteresis phenomena, and propose their meaningful generalizations. We consider DAEs of index 2 having two features: i) non-linearity of hysteresis type modeled by a sweeping process, and ii) impulsive control represented by a bounded signed Borel measure. For such a DAE, we design an equivalent structural form, based on the Kronecker-Weierstrass transformation, and prove a necessary and sufficient condition for the existence and uniqueness of a solution to an initial value problem. We propose a notion of generalized solution to a DAE as a realization of impulsive trajectory relaxation. This relaxation is described by a dynamical system with states of bounded variation and can be equivalently represented as a system of "ordinary" DAEs.
\end{abstract}

\section{Key words}

Differential-algebraic equations, hysteresis, sweeping process, impulsive control, measure-driven dynamical systems.

\section{Introduction}

Differential-algebraic equations (DAEs) is a wellrecognized and extensively studied area of the modern applied mathematics, arisen as a natural generalization of the concept of ODE. An interest to the framework of DAEs was attracted by multiple applications in rigidbody dynamics with friction and hybrid control engineering (see, e.g. [Brogliato, 1999, Song, Krauss, Kumar and Dupont, Stewart, 2000, Heemels, Schumacher, and Weiland, 2000]), as well as in modeling of electric circuits with hysteresis phenomena [Adly, Haddad, and
Thibault, 2014, Acary, Bonnefon, and Brogliato, 2011].

In this paper, we investigate and promote a very specific and poorly-studied class of DAEs, where hysteresis phenomena, represented through sweeping processes [Kunze and Marques, 2000, Moreau, 1977], are combined with impulsive behavior of the modeled dynamic process. The main motivation for this study is due to the above mentioned models of electric circuits, where impulses do naturally appear, see, e.g., [Acary, Bonnefon, and Brogliato, 2011].

\section{DAEs with hysteresis}

Consider the following DAE of index 2 paired with a sweeping process of a rate independent hysteresis type (modeled by the play operator [Brokate and Sprekels, 1996, Krejčí, 1991]):

$$
\begin{gathered}
A \dot{x}(t)=B x(t)+C y(t), \quad x\left(t_{0}\right)=x_{0}, \\
-\ddot{y}(t) \in \mathcal{N}_{Q(t)}(\dot{y}(t)), \\
y\left(t_{0}\right)=y_{0}, \quad \dot{y}\left(t_{0}\right)=y_{1} \in Q\left(t_{0}\right) .
\end{gathered}
$$

Here, $T=\left[t_{0}, t_{1}\right]$ is a given time interval, $x(t), y(t) \in$ $\mathbb{R}^{n} ; Q(t)=x(t)-Z$ is a "moving set", where $Z$ is a given closed convex subset of $\mathbb{R}^{n}$, and $\mathcal{N}_{Q}(u)$ denotes the normal cone to a closed convex set $Q$ at a point $u$; $A, B, C \in \mathbb{R}^{(n \times n)}$ are given constant matrices, wherein $A$ is singular, while the pair $(A, B)$ is assumed to be regular. Recall that a pair $(A, B)$ of matrices is called regular, if there exists $\lambda \in \mathbb{R}^{n}$ such that $\operatorname{det}(\lambda A-B) \neq$ 0.

By a solution of (1), (2) we mean a pair of absolutely continuous $\left(W^{1,1}\right)$ functions $(x, y)$ satisfying (1), (2) for almost all (a.e.) $t \in T$ with respect to (w.r.t.) the usual Lebesgue measure. 
As is known [Gantmacher, 1988], for any regular pair $(A, B)$, there exist nonsingular matrices $M, P$ such that

$$
M A P^{-1}=\left(\begin{array}{cc}
E_{k} & 0 \\
0 & N
\end{array}\right), M B P^{-1}=\left(\begin{array}{cc}
J & 0 \\
0 & E_{n-k}
\end{array}\right),
$$

where $J$ has the Jordan canonical form, and $N$ is a nilpotent matrix of index $l$ (upper triangular matrix with $l$ square null blocks on diagonal, such that $N^{l}=0$ ) also having the Jordan form.

We assume that matrix $N$ is nilpotent of index 2 . Then, system (1), (2) rewrites:

$$
\begin{gathered}
\dot{z}_{1}(t)=J z_{1}(t)+G_{1} y(t), \quad z\left(t_{0}\right)=P x_{0}, \\
z_{2}(t)=-G_{2} y(t)-N G_{2} \dot{y}(t), \\
-\ddot{y}(t) \in \mathcal{N}_{\mathcal{Q}(t)}(\dot{y}(t)), \\
y\left(t_{0}\right)=y_{0}, \quad \dot{y}\left(t_{0}\right)=y_{1} \in \mathcal{Q}\left(t_{0}\right),
\end{gathered}
$$

where $z(t) \doteq P x(t)=\left(z_{1}(t), z_{2}(t)\right), z_{1}(t) \in \mathbb{R}^{k}$, $z_{2}(t) \in \mathbb{R}^{n-k}, M C=\left(G_{1}, G_{2}\right)$, and $\mathcal{Q}(t) \doteq$ $P^{-1} z(t)-Z$.

The following assertion gives a necessary and sufficient condition for the existence of a unique solution to system (1), (2) (or rather (3)-(5)).

Proposition 2.1. Let $(A, B)$ be regular. Then problem (1), (2) has a unique solution on $T$ iff

$$
\left(\begin{array}{cc}
0 & 0 \\
0 & E_{n-k}
\end{array}\right) P x_{0}=\left(\begin{array}{c}
0 \\
-G_{2} y_{0}-N G_{2} y_{1}
\end{array}\right) .
$$

The proof is straightforward:

1) Suppose that $(A, B)$ is regular, and problem (1), (2) has a unique solution on $T$. The change of variables $x(t)=P^{-1}\left(z_{1}(t), z_{2}(t)\right)$ reduces (1), (2) to (3)-(5), and (6) immediately follows for $t=t_{0}$.

2) Assume that $(A, B)$ is regular, and (6) holds. Denote $\mathbf{x} \doteq\left(z_{1}, y, \dot{y}\right), \mathbf{f}(\mathbf{x}) \doteq\left(J z_{1}, \dot{y}, 0 \in \mathbb{R}^{n}\right), \mathbf{Q}(t) \doteq$ $\mathbb{R}^{k+n} \times \mathcal{Q}(t), t \in T$, and rewrite (3), (5) as a perturbed sweeping process

$$
-\dot{\mathbf{x}}+\mathbf{f}(\mathbf{x}) \in \mathcal{N}_{\mathbf{Q}(t)}(\mathbf{x}) .
$$

Note that the set $\mathbf{Q}(t)$ is closed and convex for all $t \in T$. Furthermore, function $\mathbf{f}$ is linear. Then, the existence and uniqueness of a solution to an initial value problem for system (7) follows from the "catching-up" algorithm for perturbed sweeping processes [Adly, Haddad, and Thibault, 2014]. It remains to agree the value $z_{2}\left(t_{0}\right)$ provided (3) and (4), which is given by condition (6).

Remark 2.1. Let $Q(t) \doteq x(t)-Z$ with $x \in W^{1,1}$. Since the addressed sweeping process is defined through a play operator, differential inclusion

$$
\begin{aligned}
-\dot{u}(t) & \in \mathcal{N}_{Q(t)}(u(t)), \\
u\left(t_{0}\right) & =u_{0} \in Q\left(t_{0}\right),
\end{aligned}
$$

is equal to the following differential variational inequality:

$$
\left\{\begin{array}{lc}
\langle\rho-x(t)+u(t), \dot{u}(t)\rangle \leq 0 & \forall \rho \in Z, \text { a.e. } t \in T, \\
x(t)-u(t) \in Z, & \forall t \in T .
\end{array}\right.
$$

Example 1: Modeling of an Electric Circuit by a Differential-algebraic Sweeping Process Consider an electric circuit involving six resistors, three inductors, two ideal diodes, and a current source $c(t)$ (see Fig. 1). Recall that the ideal diode's voltage/current law is described by the complementary condition

$$
0 \leq-V_{d}(t) \perp i(t) \geq 0,
$$

where $V_{d}$ denotes the potential across the diode and $i$ is the current through the diode. This complementary condition can be reformulated as an inclusion

$$
V_{d}(t) \in \mathcal{N}_{\mathbb{R}_{+}}(i(t)),
$$

where $\mathbb{R}_{+} \doteq[0,+\infty)$. Let $R_{k}, L_{k}>0, k=1,2,3$, denote the load resistances and the inductors, respectively. Then the Kirchhoff's law writes:

$$
\begin{aligned}
& L_{1} \dot{x}_{1}+R_{1} x_{1}+R_{2}\left(x_{1}-x_{2}\right)+V_{d 1}=0, \\
& R_{1} x_{2}-R_{2}\left(x_{1}-x_{2}\right)=0, \\
& L_{2} \dot{x}_{3}+R_{1} x_{3}+R_{3}\left(x_{3}-x_{4}\right)=0, \\
& L_{3} \dot{x}_{4}+R_{1} x_{4}-R_{3}\left(x_{3}-x_{4}\right)+V_{d 2}=0 .
\end{aligned}
$$

Setting

$$
\begin{gathered}
x=\left(\begin{array}{l}
x_{1} \\
x_{2} \\
x_{3} \\
x_{4}
\end{array}\right), \quad A=\left(\begin{array}{cccc}
L_{1} & 0 & 0 & 0 \\
0 & 0 & 0 & 0 \\
0 & 0 & L_{2} & 0 \\
0 & 0 & 0 & L_{3}
\end{array}\right), \\
B=\left(\begin{array}{cccc}
R_{1}+R_{2} & -R_{2} & 0 & 0 \\
R_{2} & -\left(R_{1}+R_{2}\right) & 0 & 0 \\
0 & 0 & R_{1}+R_{3} & -R_{3} \\
0 & 0 & -R_{3} & R_{1}+R_{3}
\end{array}\right),
\end{gathered}
$$

relations (8)-(11) take the form of a differentialalgebraic sweeping process (DASP):

$$
A \dot{x}(t)+B x(t) \in-\mathcal{N}_{C(t)}(x(t)),
$$

where $C(t)=[c(t),+\infty) \times \mathbb{R}^{2} \times \mathbb{R}_{+}$is a moving set.

If $c(\cdot)$ is Lipschitz continuous, one can show that DASP (12) has a unique solution for any initial condition $x(0)=x_{0} \in C(0)$ satisfying the relation

$$
x_{10}=\left(\frac{R_{1}}{R_{2}}+1\right) x_{20} .
$$

Indeed, equality (9) gives: $x_{2}=\frac{R_{2} x_{1}}{R_{1}+R_{2}}$. Putting this 


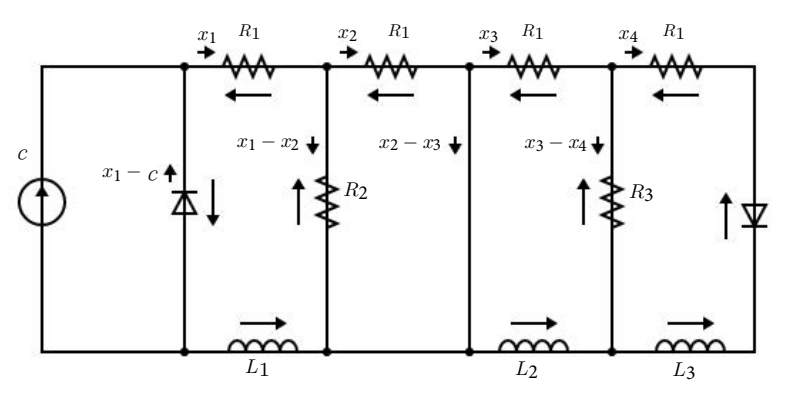

Figure 1. Example 1: Electric circuit with resistors, inductors and ideal diodes.

expression into (8), we obtain

$$
\begin{array}{r}
\dot{x}_{1}(t)+a x_{1}(t) \in-\mathcal{N}_{[c(t),+\infty)}\left(x_{1}(t)\right), \\
x_{1}(0)=x_{10} \in[c(0),+\infty),
\end{array}
$$

where $a=\frac{1}{L_{1}}\left(R_{1}+\frac{R_{1} R_{2}}{R_{1}+R_{2}}\right)$. Now, denote $\zeta=$ $\left(x_{3}, x_{4}\right)$ and rewrite (10), (11) as follows:

$$
\begin{array}{r}
\dot{\zeta}(t)+b \zeta(t) \in-\mathcal{N}_{\mathbb{R} \times \mathbb{R}_{+}}(\zeta(t)), \\
\zeta(0)=\zeta_{0} \in \mathbb{R} \times \mathbb{R}_{+}, \\
b=\left(\begin{array}{cc}
\frac{R_{1}+R_{3}}{L_{2}} & -\frac{R_{3}}{L_{2}} \\
-\frac{R_{3}}{L_{3}} & \frac{R_{1}+R_{3}}{L_{3}}
\end{array}\right) .
\end{array}
$$

It remains to note that (14), (15) are perturbed sweeping processes, and each of them has a unique solution for given $x_{0}$ and $c(\cdot)$ [Adly, Haddad, and Thibault, 2014].

Note that the current source in Example 1 can be described by a discontinuous control function $c(\cdot)$. In this case, jumps of the moving set $C(t)$ produce jumps of the respective state solution, which leads to a concept of impulsive DAE with hysteresis.

Example 2 Consider a DAE of the form (1), (2) with

$$
\begin{gathered}
A=\left(\begin{array}{ccc}
1 & 0 & -1 \\
0 & 0 & -1 \\
0 & 0 & 0
\end{array}\right), \quad B=\left(\begin{array}{ccc}
2 & -1 & -2 \\
0 & -1 & 2 \\
0 & 0 & 1
\end{array}\right) \\
C=\left(\begin{array}{ccc}
1 & -1 & 0 \\
2 & 0 & -1 \\
0 & 0 & 0
\end{array}\right)
\end{gathered}
$$

paired with the differential inclusion (2) driven by the moving set $Q(t)=x(t)-\mathbf{B}_{1}$, where $\mathbf{B}_{1}$ is the closed unit ball in $\mathbb{R}^{3}$, under initial conditions

$$
\begin{aligned}
& x\left(t_{0}\right)=(0.5,0.7,0), \\
& y\left(t_{0}\right)=(0.3,0.7,-0.1), \\
& \dot{y}\left(t_{0}\right)=(0.7,0.3,0.1) .
\end{aligned}
$$

As is clear, the pair $(A, B)$ is regular. Then there exist nonsingular matrices

$$
M=\left(\begin{array}{ccc}
1 & -1 & 4 \\
0 & -1 & 2 \\
0 & 0 & 1
\end{array}\right), \quad P=\left(\begin{array}{lll}
1 & 0 & 0 \\
0 & 1 & 0 \\
0 & 0 & 1
\end{array}\right)
$$

such that

$$
\begin{gathered}
M A P^{-1}=\left(\begin{array}{cc}
E_{k} & 0 \\
0 & N
\end{array}\right)=\left(\begin{array}{c|cc}
1 & 0 & 0 \\
\hline 0 & 0 & 1 \\
0 & 0 & 0
\end{array}\right) ; \\
M B P^{-1}=\left(\begin{array}{cc}
J & 0 \\
0 & E_{n-k}
\end{array}\right)=\left(\begin{array}{c|cc}
2 & 0 & 0 \\
\hline 0 & 1 & 0 \\
0 & 0 & 1
\end{array}\right) .
\end{gathered}
$$

The system thus reduces to

$$
\begin{gathered}
\dot{z}_{1}(t)=2 z_{1}(t)+\left(\begin{array}{ccc}
-1 & -1 & 1
\end{array}\right) y(t), \\
z_{2}(t)=\left(\begin{array}{ccc}
2 & 0 & -1 \\
0 & 0 & 0
\end{array}\right) y(t),
\end{gathered}
$$

where $\left(z_{1}(t), z_{2}(t)=\left(z_{2}^{1}, z_{2}^{2}\right)(t)\right)=P x(t)=x(t)$.

By Proposition 2.1, we see that relation (6) does hold. Hence, problem (1), (2), (16)-(18) has a unique solution on any finite interval $T=\left[t_{0}, t_{1}\right], t_{1}>t_{0}$.

A typical trajectory of this DAE, in its equivalent form (3)-(5), is presented on Fig. 2.

\section{DAEs with Hysteresis and Control Measures}

Now, we assume that dynamic processes, model by system (1), (2), can be externally controlled; furthermore, control influences are admitted to have "shock", impulsive nature (e.g., in the spirit of [Acary, Bonnefon, and Brogliato, 2011]).

Consider a generalization of (1), (2), where the part of control inputs is played by bounded Borel measures:

$$
\begin{gathered}
A d x(t)=d(B x(t)+C y(t)+D h(t)), \\
\dot{h}(t)=w(t), \quad d w(t)=\mu(d t),
\end{gathered}
$$

$$
\begin{gathered}
x\left(t_{0}\right)=x_{0}, \quad h\left(t_{0}\right)=h_{0}, \quad w\left(t_{0}\right)=w_{0}, \\
-d \dot{y}(t) \in \mathcal{N}_{Q(t)}(\dot{y}(t)), \\
y\left(t_{0}\right)=y_{0}, \quad \dot{y}\left(t_{0}\right)=y_{1} \in Q\left(t_{0}\right), \\
\mu \in C^{*}(T, K) .
\end{gathered}
$$

Here, the moving set $Q(t)$ is defined as above, $K$ is a closed convex cone in $\mathbb{R}^{m}, h(t), w(t) \in \mathbb{R}^{m}, D \in$ $\mathbb{R}^{(n \times m)}$ is given constant matrix. Though impulsive controls - measures $\mu-$ do not appear in (19) explicitly, the DAE-structure of the model leads to the appearance of discontinuous states with bounded total variation on $T$ ( $B V$-solutions). In other words, functions 


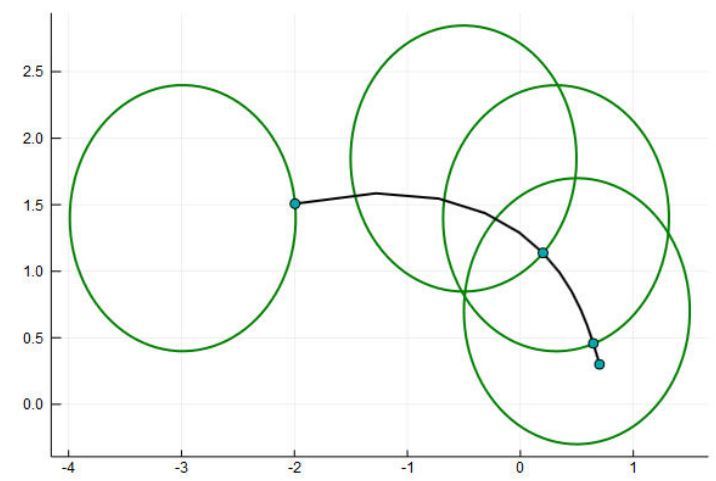

Figure 2. Example 2: Sweeping of a point by the moving set on the plane $\left(z_{1}, z_{2}^{1}\right)$.

$x(\cdot), \dot{y}(\cdot), w(\cdot)$ can jump, and system (19)-(24) should be considered within the class of impulsive controls and $B V$-solutions (the notion of $B V$-solution for the sweeping process (22) is given in [Samsonyuk and Timoshin, 2018]).

System (19)-(24) can be interpreted as an impulsivetrajectory relaxation of the following conventional DAE with hysteresis:

$$
\begin{gathered}
A \dot{x}(t)=B x(t)+C y(t)+D h(t), \\
\dot{h}(t)=w(t), \quad \dot{w}(t)=v(t), \\
x\left(t_{0}\right)=x_{0}, \quad h\left(t_{0}\right)=h_{0}, \quad w\left(t_{0}\right)=w_{0}, \\
-\ddot{y}(t) \in \mathcal{N}_{Q(t)}(\dot{y}(t)), \\
y\left(t_{0}\right)=y_{0}, \quad \dot{y}\left(t_{0}\right)=y_{1} \in Q\left(t_{0}\right), \\
v(t) \in K \text { for a.e. } t \in T,
\end{gathered}
$$

where $x(\cdot), \dot{y}(\cdot)$, and $w(\cdot)$ are yet of class $W^{1,1}$. Again, similar to (3)-(5), system (25)-(28) could be rewritten as follows:

$$
\begin{gathered}
\dot{z}_{1}(t)=J z_{1}(t)+G_{1} y(t)+F_{1} h(t), \\
z_{2}(t)=-G_{2}(y(t)+N \dot{y}(t))-F_{2}(h(t)+N w(t)), \\
\dot{h}(t)=w(t), \quad \dot{w}(t)=v(t), \\
z\left(t_{0}\right)=P x_{0}, \quad h\left(t_{0}\right)=h_{0}, \quad w\left(t_{0}\right)=w_{0}, \\
-\ddot{y}(t) \in \mathcal{N}_{\mathcal{Q}(t)}(\dot{y}(t)), \\
y\left(t_{0}\right)=y_{0}, \quad \dot{y}\left(t_{0}\right)=y_{1} \in \mathcal{Q}\left(t_{0}\right), \\
v(t) \in K \text { for a.e. } t \in T,
\end{gathered}
$$

where $z(t)=P x(t)=\left(z_{1}(t), z_{2}(t)\right), M C=$ $\left(G_{1}, G_{2}\right), M D=\left(F_{1}, F_{2}\right), z_{1}(t) \in \mathbb{R}^{k}, z_{2}(t) \in \mathbb{R}^{n-k}$, and $\mathcal{Q}(t)=P^{-1} z(t)-Z$.

Let $\left(z_{10}, z_{20}\right) \doteq P x_{0}$. Then, given $\mu$, the initial condition guaranteeing the existence and uniqueness of a solution for system (25)-(30) takes the form:

$$
z_{20}=-G_{2} y_{0}-N G_{2} y_{1}-F_{2} h_{0}-N F_{2} w_{0} .
$$

By the arguments [Samsonyuk and Timoshin, 2018] and the singular space-time transformation [Miller, 1996, Miller and Rubinovich, 2013, Sesekin and Zavalishchin, 1997], impulsive system (19)-(24) can be transformed to a continuous (non-impulsive) system:

$$
\begin{gathered}
\eta^{\prime}(\tau)=\omega_{0}(\tau), \eta(0)=t_{0}, \eta\left(\tau_{1}\right)=t_{1}, \\
\xi_{1}^{\prime}(\tau)=\left(J \xi_{1}(\tau)+G_{1} \nu(\tau)+F_{1} \zeta(\tau)\right) \omega_{0}(\tau), \\
\xi_{2}(\tau)=-G_{2}(\nu(\tau)+N \dot{\nu}(\tau))-F_{2}(\zeta(\tau)+N \gamma(\tau)), \\
\zeta^{\prime}(\tau)=\gamma(\tau) \omega_{0}(\tau), \gamma^{\prime}(\tau)=\omega(\tau), \\
\xi(0)=P x_{0}, \quad \zeta(0)=h_{0}, \quad \gamma(0)=w_{0}, \\
-\nu^{\prime \prime}(\tau) \in \mathcal{N}_{\mathcal{C}(\tau)}\left(\nu^{\prime}(\tau)\right), \\
\nu(0)=y_{0}, \quad \nu^{\prime}(0)=y_{1} \in \mathcal{C}(0), \\
\left(\bar{\omega}_{0}(\tau), \bar{\omega}(\tau)\right) \in \operatorname{co} \tilde{K}_{1} \text { for a.e. } \tau \in\left[0, \bar{\tau}_{1}\right]
\end{gathered}
$$

where prime denotes the derivative w.r.t. the new time variable $\tau, \mathcal{C}(\tau) \doteq P^{-1} \xi(\tau)-Z, \tilde{K}_{1} \doteq\left\{\left(\omega_{0}, \omega\right) \in\right.$ $\left.[0,1] \times K \mid \omega_{0}+\|\omega\|_{1}=1\right\},\|\cdot\|_{1}$ is the Manhattan norm in $\mathbb{R}^{n}$, and co $A$ is the convex hull of a set $A$.

The transformed system is extended to a new time scale $\tau$, where variables $(t, x, y, h, w)$ become $(\eta, \xi, \nu, \zeta, \gamma)$, while control measures $\mu$ are represented by bounded measurable functions $\omega_{0}(\cdot), \omega(\cdot)$. The auxiliary system describes $B V$-solutions of (19)-(24) up to an appropriate discontinuous time change.

\section{Further Generalizations and Concluding Re- marks}

In this section, we shall propose some promising generalizations of the considered DAEs, and mark out natural direction of the future work.

The models, overviewed in the previous sections, can be reformulated under the framework of so-called differential variational inequalities (DVIs) [Pang and Stewart, 2008]. Such a fact is not surprising in the nonimpulsive setup, where DVIs are known to cover a plenty of dynamic complementarity problems, sweeping processes, and differential algebraic equations [Adly, Haddad, and Thibault, 2014, Brokate, Krejčí, and Schnabel, 2004, Brokate and Sprekels, 1996, Facchinei and Pang, 2003, Krejčí, 1991, Moreau, 1977].

A natural extension of the impulsive model, addressed in the previous section, then leads to the concept of measure-driven differential variational inequality (MDVI), which is a combination of a measure differential equation, a finite-dimensional variational inequality, and/or a differential quasi-variational inequality. MDVIs are studied in concern with rate independent hysteresis processes, and sweeping processes in [Kopfova and Recupero, 2016, Recupero, 2011, Recupero, 2015, Samsonyuk and Timoshin, 2018, Samsonyuk and Timoshin, 2019]. 
A class of such dynamical systems, which could be a challenging object of our further study, formally looks as follows:

$$
\begin{gathered}
d x(t)=f(t, x, y) d t+G(t, x, y) \mu(d t), \\
\mu \in C^{*}(T, K), \\
\langle F(t, x(t), y(t)), \rho-y(t)\rangle \geq 0 \\
\text { for all } \rho \in Z \text { and a.e. } t \in T .
\end{gathered}
$$

Here, $Z$ is a given closed convex subset of $\mathbb{R}^{k}, F$ is a given map of a certain appropriate type, $K$ is a closed convex cone in $\mathbb{R}^{m}$, and $x(\cdot)$ and $y(\cdot)$ are functions of bounded variation.

Note that (31), (32) can be regarded as a relaxation of the non-impulsive DVI

$$
\begin{gathered}
\dot{x}=f(t, x, y)+G(t, x, y) v, \\
v(t) \in K \text { for a.e. } t \in T, \\
\langle F(t, x(t), y(t)), \rho-y(t)\rangle \geq 0 \\
\quad \text { for all } \rho \in Z \text { and } t \in T,
\end{gathered}
$$

with absolutely continuous trajectories $x(\cdot), y(\cdot)$ and "usual" control $v(\cdot) \in L^{\infty}\left(T, \mathbb{R}^{m}\right)$, and can be treated by the standard singular space-time transformation [Karamzin, Oliveira, Pereira, and Silva, 2015, Miller, 1996, Miller and Rubinovich, 2013, Sesekin and Zavalishchin, 1997].

We also remark that, for $Z=\mathbb{R}^{k}$ and $F: T \times \mathbb{R}^{n} \times$ $\mathbb{R}^{m} \rightarrow \mathbb{R}^{m}$, DVI (34) boils down to

$$
F(t, x(t), y(t))=0,
$$

while (33), (34) turns into a DAE.

As a concluding remark, we mention that the concept of MDVI can be extended to impulsive control systems paired with another type of complementarity conditions, namely the "orthogonality" between the driving measure and an input, depending on the one-sided limits of the state. Such systems seem to be a meaningful formalization of mechanical systems with blockable degrees of freedom, and are recently studied in [Goncharova and Staritsyn, 2017, Goncharova and Staritsyn, 2018, Staritsyn and Goncharova, 2019].

\section{Acknowledgements}

The work is partially supported by the Russian Foundation for Basic Research, projects nos 18-31-20030, 1801-00026.

\section{References}

Adly, S., Haddad, T., and Thibault, L. (2014). Convex sweeping process in the framework of measure differential inclusions and evolution variational inequalities. Mathematical Programming B, Springer, 148(1), pp. 5-47.
Acary, V., Bonnefon, O., and Brogliato, B. (2011). Nonsmooth Modelling and Simulation for Switched Circuits. Springer Science+Business Media B.V.

Brogliato, B. (1999). Nonsmooth Mechanics. Models, Dynamics and Control. Springer, London.

Brokate, M., Krejčí, P., and Schnabel, H. (2004). On uniqueness in evolution quasivariational inequalities. $J$. Convex Anal., 11, pp. 111-130.

Brokate, M. and Sprekels, J. (1996). Hysteresis and Phase Transitions. Ser. Appl. Math. Sci., vol. 121. Springer-Verlag, New York.

Gantmacher, F.R. (1988). The Theory of Matrices. Nauka, Moscow (in Russian).

Goncharova, E. and Staritsyn, M. (2017). Relaxation and optimization of impulsive hybrid systems: Inspired by impact mechanics. In Proceedings of the 14th International Conference on Informatics in Control, Automation and Robotics, 1, pp. 474-485.

Goncharova, E. and Staritsyn, M. (2018). On BVextension of asymptotically constrained control-affine systems and complementarity problem for measure differential equations. Discrete and Continuous Dynamical Systems-Series S., 11(6), pp. 1061-1070.

Facchinei, F. and Pang, J.S. (2003). Finite-Dimensional Variational Inequalities and Complementarity Problems. Springer, New York.

Heemels, W.P.M.H., Schumacher, J.M., and Weiland, S. (2000). Linear complementarity systems. SIAM J. Appl. Math., 60, pp. 1234-1269.

Karamzin, D.Yu., Oliveira, V.A., Pereira, F.L., and Silva, G.N. (2015). On the properness of the extension of dynamic optimization problems to allow impulsive controls. ESAIM: Control, Optimisation and Calculus of Variations, 21(3), pp. 857-875.

Kopfova, J. and Recupero, V. (2016). BV-norm continuity of sweeping processes driven by a set with constant shape. J. Differ. Equations, 261(10), pp. 5875-5899.

Krejčí, P. (1991). Vector hysteresis models. European J. Appl. Math., 2, pp. 281-292.

Kunze, M. and Marques, M.D.M. (2000). An Introduction to Moreau's Sweeping Process. In Brogliato B. (eds) Impacts in Mechanical Systems. Lecture Notes in Physics, 551. Springer, Berlin, Heidelberg.

Miller, B. (1996). The generalized solutions of nonlinear optimization problems with impulse control. SIAM J. Control Optim., 34, pp. 142-440.

Miller, B.M. and Rubinovich E.Ya. (2013). Discontinuous solutions in the optimal control problems and their representation by singular space-time transformations. Autom. Remote Control, 74, pp. 1969-2006.

Moreau, J.-J. (1977). Evolution problem associated with a moving convex set in a Hilbert space. J. Differential Eq., 26, pp. 347-374.

Pang, J.S. and Stewart, D.E. (2008). Differential variational inequalities. Math. Progam. Ser. A., 113, pp. 345-424. 
Recupero, V. (2011). BV solutions of the rate independent inequalities. Ann. Scuola Norm. Sup. Pisa Cl. Sci., $\mathbf{X}(5)$, pp. 269-315.

Recupero, V. (2015). BV continuous sweeping processes. J. Differential Equation, 259, pp. 4253-4272.

Samsonyuk, O.N. and Timoshin, S.A. (2018). BV solutions of rate independent processes driven by impulsive controls. IFAC-Papers OnLine, 51(32), pp. 361-366.

Samsonyuk, O.N. and Timoshin, S.A. (2019). Optimal control problems with states of bounded variation and hysteresis. Journal of Global Optimization, 74(3), pp. 565-596.
Sesekin, A. and Zavalishchin, S. (1997). Dynamic Impulse Systems: Theory and Applications. Kluwer Academic Publishers, Dordrecht.

Song, P., Krauss, P., Kumar, V., Dupont, P. (1999). Analysis of rigid-body dynamic models for simulation of systems with frictional contacts. J. Appl. Mech., 68, pp. 118-128.

Staritsyn, M. and Goncharova, E. (2019). On complementarity measure-driven dynamical systems, Lecture Notes in Electrical Engineering, 495, pp. 699-718.

Stewart, D.E. (2000). Rigid-body dynamics with friction and impact. SIAM Rev., 42, pp. 3-39. 